\title{
Students' strategies for solving a multirepresentational partial derivative problem in thermodynamics
}

\author{
Rabindra R. Bajracharya, ${ }^{1, *}$ Paul J. Emigh, ${ }^{2}$ and Corinne A. Manogue ${ }^{2}$ \\ ${ }^{1}$ Chemical and Physical Sciences Department, Missouri Southern State University, \\ 3950 E. Newman Rd., Joplin, Missouri 64801, USA \\ ${ }^{2}$ Department of Physics, Oregon State University, 301 Weniger Hall, Corvallis, Oregon 97331, USA
}

(Received 31 January 2018; revised manuscript received 5 May 2019; published 5 September 2019)

\begin{abstract}
We present an empirical analysis of students' use of partial derivatives in the context of problem solving in upper-division thermodynamics. Task-based individual interviews were conducted with eight middledivision physics students. The interviews involved finding a partial derivative from information presented in a table and a contour plot. Using thematic analysis, we classified student problem-solving strategies into two principal categories: the analytical derivation strategy and the graphical analysis strategy. We developed a new flowchartlike analysis method: representational transformation. Our analysis of students' strategies using this method revealed three types of transformation phenomena: translation, consolidation, and dissociation. Students in this study did not seem to have much difficulty with the concepts underlying the partial derivative; instead, they seemed to have difficulty with the transformation phenomena, particularly the consolidation of multiple representations into a single representation.
\end{abstract}

DOI: 10.1103/PhysRevPhysEducRes.15.020124

\section{INTRODUCTION}

Mathematics serves not only as a language to express physical laws and phenomena in clear and concise ways, but also as a logical pathway for solving problems. The use of mathematical concepts and procedures is thus inevitable in physics problem solving. One of the important mathematical concepts commonly used in upper-division physics, particularly in thermodynamics, is the partial derivative. Partial derivatives are useful for representing and interpreting thermodynamic processes and properties, such as the specific heat capacity at constant volume, $C_{V}=(\partial U / \partial T)_{V}$. An important set of tools for relating partial derivatives are various chain rules, which can help express unmeasurable quantities, like entropy and internal energy, in terms of quantities that are measurable.

Physics problems can often be solved using more than one strategy and using different sets of mathematical tools and different representations of information. To solve thermodynamics problems in multiple representations, students need deep conceptual understanding and representational fluency in addition to procedural skills. Although it is possible to present problems involving partial derivatives using various representations-such as symbolic [e.g., $\left.(\partial P / \partial V)_{T}\right]$,

\footnotetext{
*bajracharya-r@mssu.edu

Published by the American Physical Society under the terms of the Creative Commons Attribution 4.0 International license. Further distribution of this work must maintain attribution to the author(s) and the published article's title, journal citation, and DOI.
}

geometric (3D surfaces), graphical (contour maps), and numerical (data) - many traditional thermodynamic problems exclusively involve symbolic manipulation, also known as analytical derivation [1]. We define analytical derivation as a process by which an unknown physical quantity is expressed in terms of known physical quantities as an equation using relevant mathematical and physical knowledge.

A robust understanding of partial derivatives requires some or all of the following concepts:

(a) Conceptual: A partial derivative represents the rate of change in a function that depends on two or more variables due to a change in only one variable while holding all other independent variables constant. In thermodynamics, especially, it is important to identify which variables are independent before taking a partial derivative.

(b) Procedural: A partial derivative can be evaluated numerically by computing the ratio of small changes in the function and the variable holding all other independent variables constant.

(c) The chain rule: A partial derivative can be expressed in terms of one or more other partial derivatives using mathematical identities called chain rules.

(d) Representational: A partial derivative can be represented in multiple representations, such as symbolic, graphical, and numerical (tabular). Partial derivatives can be computed (sometimes approximately) from information in these various representations.

Previous studies and our own anecdotal evidence reveal that students often struggle with one or more of the above 
aspects even when they are otherwise able to solve many traditional homework problems [2-4]. We find that the students in this study seem to have more difficulties with the representational aspect than with the other aspects. In this article, we report results from part of a long-term ongoing research project focused on the teaching and learning of partial derivatives [5-14]. One result of this project has been the development of several activities and pedagogical approaches aimed at improving the teaching and learning of various mathematical concepts in thermodynamics, including partial derivatives [13-19]. In this qualitative study, our aim is to investigate students' understanding and application of partial derivatives when solving a multirepresentational problem, with the ultimate goal of informing curricular development. We note that it is not our intent to compare the performance of students who have completed different curricula, but only to explore students' ideas. We used a thermodynamics context to investigate students strategies for solving a multirepresentational partial derivative problem because of its prevalent application in thermodynamics.

In order to probe students' understanding of the partial derivative concept and their abilities to apply chain rules, we designed a multistage interview protocol. Because the students in this study experienced instruction that focused on multiple representations of partial derivatives in physics courses, we deliberately gave them a challenging multirepresentational problem intended to invoke their reasoning and problem-solving abilities. Using thematic analysis, we identified the students' strategies for solving the problem, both correct and incorrect [20,21]. Our analysis of the students' responses during the four stages of the interview revealed that even though they did not have much difficulty with the conceptual and the procedural aspects, they struggled with the chain rule and the representational aspects of partial derivatives, which is in line with previous research by our group [22].

We find that even with extensive classroom experiences involving multiple representations, students still had difficulty coordinating multiple representations. Although our initial analysis hinted that students have difficulty with multiple representations, it did not clearly reveal how the chosen representations affected students' problem solving. To help resolve this emergent question, we developed a new analysis approach focused on students' manipulation of representations.

Because the partial derivative problem given in the interviews involves multiple representations, our secondary analysis focused on how students dealt with the given representations. We call this analysis the representational transformation (RT) method. The RT method led us to discover various RT phenomena that are required to solve the given problem, such as translation from one representation to another, consolidation of multiple representations, and dissociation of information into multiple representations. Using this method, we were able to characterize how the different solution methods attempted by the students involved these various phenomena. We consider our analysis method to be useful for analyzing how students solve multirepresentational problems in other contexts. We emphasize, however, that the main purpose of this study is not to describe our analytical tool, but to investigate students' problem-solving strategies. The primary research question in this study is

(i) What strategies do students use for solving a multirepresentational partial derivative problem in a thermodynamics context?

However, the following two subsequent research questions arise as we dig deeper into the students' strategies:

(ii) How do students deal with the representations involved in the problem? and

(iii) Which steps in the representational aspects of the problem are difficult for students?

In the next section, we present a brief review of related literature on the teaching and learning of partial derivatives. In Sec. III, we briefly discuss how students at Oregon State University are taught partial derivatives, particularly in thermodynamics. In Sec. IV, we describe our overall research design, including the student population and the methodology used to collect and analyze our data. Section V presents the findings of both our analysis methods. Finally, in Sec. VI, we discuss our results and give some implications for both instructors and researchers.

\section{RELEVANT LITERATURE}

There has not been much research on the teaching and learning of partial derivatives in mathematics, chemistry, or physics education. However, a few studies on student understanding of the partial derivative in thermodynamics contexts together with considerable anecdotal experience suggest that conceptual and procedural difficulties are common [2-4].

For example, Bucy, Thompson, and Mountcastle showed that students have difficulty with partial derivative problems that involve analytical derivation [3]. They asked upperdivision students to derive $(\partial \beta / \partial P)_{T}+(\partial \kappa / \partial T)_{P}=0$, where $\beta$ and $\kappa$ were defined as the isothermal compressibility and thermal expansivity, respectively. More than half of the students were unable to derive the expression correctly. A common incorrect response was that because $T$ and $P$ are already held constant in $\kappa=-\frac{1}{V}\left(\frac{\partial V}{\partial P}\right)_{T}$ and $\beta=\frac{1}{V}\left(\frac{\partial V}{\partial T}\right)_{P}$, any successive derivative of these quantities with respect to $T$ and $P$ would yield zero. The students interpreted $(\partial \beta / \partial P)_{T}$ and $(\partial \kappa / \partial T)_{P}$ as derivatives of constant quantities.

In a separate study, Thompson, Bucy, and Mountcastle surveyed students' interpretations of partial derivative expressions with and without physics contexts [2]. They found that although students were able to give correct interpretations for the meaning of a given partial derivative, they were unable to translate a given thermodynamic process into a symbolic expression. They also found that their students could routinely derive Maxwell relations 
from given thermodynamic equations with little difficulty, but could not select an appropriate relation relevant to a given physical situation. Thompson et al. attributed these difficulties to students' lack of deep understanding of the partial derivative concept.

Becker and Towns gave a series of nine questions about partial derivatives to upper-division chemistry students [4]. The first five questions were aimed at probing students' conceptual understanding of the mathematical aspects of the partial derivative, whereas the other four questions were aimed at probing students' application of the partial derivative concept to given physical contexts. They found that the majority of the participants had a reasonable understanding of the mathematical aspects of these partial derivatives. Nearly all the students were also able to give physical interpretations of the partial derivatives in thermodynamic contexts. However, they found that the students had difficulty applying their mathematical knowledge to translate a physical situation into a symbolic expression, which corroborates the findings from the study of Thompson et al. [2].

Roundy, Kustusch, and Manogue have developed a set of activities called "Name the Experiment," intended to focus students' attention on the inverse task [13]. Each of these activities asks students to come up with a physical situation that can be represented by a given partial derivative.

Kustusch et al. studied experts' strategies for solving a partial derivative problem in a thermodynamics context [6]. They conducted task-based interviews with eight experts in physics, one in mathematics, and one in engineering. The experts were asked to derive an analytical expression for $(\partial U / \partial p)_{S}$ from given thermodynamic equations. Kustusch et al. found that the experts used a broad range of strategies: manipulating differentials, substituting terms, manipulating standard thermodynamic equations, (e.g., $d U=p d V-$ $T d S$ ), and setting the differentials of constant variables to zero (e.g., $d S=0$ ). Although these experts had been teaching thermodynamics and/or partial derivatives for several years in their respective fields, some were unable to derive the correct expression despite invoking much of the knowledge base relevant to the task. Kustusch et al.'s study shows that it is not only students but also experts who are challenged by problems involving partial derivatives in thermodynamics.

Physics and mathematics problems are often presented in various representations, such as words, pictures, equations, and graphs. Furthermore, the importance of these different representations for understanding derivatives has been highlighted by Zandieh's concept image framework [23]. We have extended this framework to include the numerical representation that has particular relevance in physics [7], and we are also in the process of extending it to encompass partial derivatives as well as ordinary derivatives [11].

Kohl and Finkelstein showed a significant difference in student performance between (almost) isomorphic problems presented in different representations (mathematical, pictorial, graphical, and verbal) [24]. In another study on the effect of representation on student problemsolving abilities, Kohl and Finkelstein found that introductory physics students' problem-solving strategies varied with representation as well as with the particular combination of representation, topic, and student knowledge base [25]. According to the authors, students who "used fewer strategies appeared to outperform those who used many." Several studies in mathematics education show that students often choose algebraic paths over visual paths for information processing, even when the former ones are more complex [26,27]. Heid claimed that students usually expect an algebraic representation when dealing with functions, and often fail to interpret graphical representations successfully [28]. Although there have been a few studies on various aspects of partial derivatives in physics and mathematics education research, we have not found any research on multiple-representational problem solving involving partial derivatives.

\section{THERMODYNAMICS AT OREGON STATE UNIVERSITY}

In physics, students generally work with partial derivatives in upper-division physics courses, most commonly in thermodynamics and electromagnetism. In this section, we briefly describe the reformed thermodynamics course called Energy and Entropy taught at Oregon State University (OSU) and discuss how students deal with partial derivatives in the course. Students typically take the Energy and Entropy course during their junior year. It is a part of the middle-level sequence of physics courses designed by the Paradigms in Physics group at OSU. This course addresses common student learning issues in thermodynamics that have been identified by researchers and thermodynamics instructors [29].

Prior to taking this course, students will typically have taken a two-year lower-division calculus sequence which includes two quarters of multivariable or vector calculus and one quarter of ordinary differential equations. A prerequisite middle-division physics course on electricity and magnetism, taken by all students, covers geometric combinations of partial derivatives - gradient, divergence, and curl.

Energy and Entropy is a nontraditional thermodynamics course that involves numerous active-engagement strategies including small white-board questions, lab activities, and structured group problem solving [15-17]. The course includes a week-long mathematics session, during which students learn the mathematics concepts that are most relevant to the course. A main feature of this session is that the mathematical concepts relevant to Energy and Entropy are introduced using multiple representations through various student-centered activities. Some activities have been discussed in detail in prior publications 
$[13,18,19,29,30]$. The specific activities included during each term depend on the instructor; below, we describe those experienced by our interviewees that are directly relevant to this research.

\section{A. Partial derivative machine activities}

The partial derivative machine is a mechanical tool that allows students to explore a wide variety of mathematics and physics concepts in a novel and hands-on context. OSU has used it for content such as numerical integration, numerical differentiation, exploration of partial derivative concepts, and development of analogies to cyclic processes in thermodynamics. A description of the partial derivative machine has previously been published $[7,19]$.

The partial derivative machine consists of a system of a spring and strings mounted on a wooden board, as shown in Fig. 1. The purpose of the string connections is to create a nonlinear system. The two ends of the spring system are connected to weight hangers via strings through pulleys on the right and the left sides of the mount board. The position of either string $\left(x_{L}\right.$ and $\left.x_{R}\right)$ can be measured using movable flags or fixed by screwing down the string. The weight on each hanger $\left(F_{L}\right.$ and $\left.F_{R}\right)$ can be varied by adding or removing masses.

During the mathematics session associated with Energy and Entropy relevant to this study, students were asked to find the partial derivative $\left(\partial x_{L} / \partial F_{L}\right)_{x_{R}}$ using the machine. The activity is designed to be open ended and exploratory, so students are guided to collect data, organize it into a table, and use the data from the table to calculate the desired partial

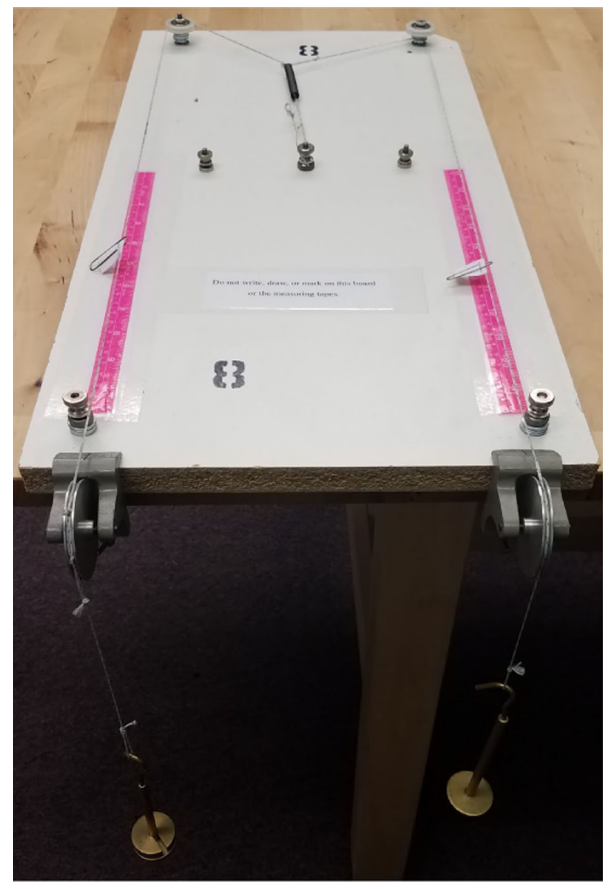

FIG. 1. The partial derivative machine, a mechanical analogue of thermodynamic systems used as a pedagogical tool.

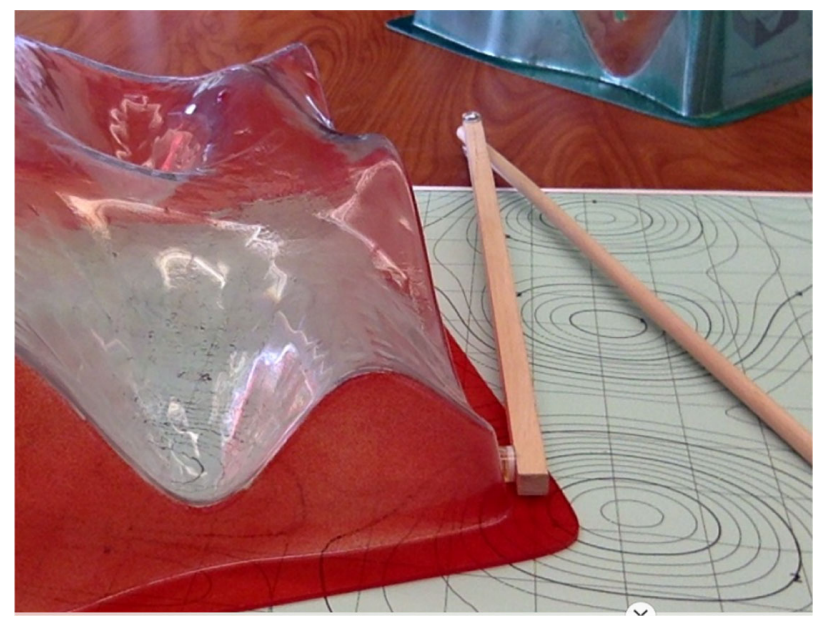

FIG. 2. A plastic surface and matching contour map used in a variety of activities aimed at improving students' understanding of multivariable calculus.

derivative. In this case, students can change $F_{L}$ by adding or removing masses while holding $x_{R}$ fixed, then find the partial derivative at various points by taking the ratio $\Delta x_{L} /\left.\Delta F_{L}\right|_{x_{R}}$ for sufficiently small changes in the force $F_{L}$.

\section{B. Surface and contour map activities}

In surface and contour map activities, students conceptualize various multivariable calculus concepts, such as definite integrals and partial derivatives, using threedimensional transparent plastic surfaces, corresponding contour maps, and a simple slope measuring tool, designed by the surfaces project team, as shown in Fig. $2[31,32]$. Participants in this study had experience with these plastic surfaces in prior upper-level physics courses on electromagnetism. In one particular activity, students are asked to find $(\partial h / \partial x)_{y}$ at a given point on a 3D surface, where $h$ is the height of the surface and $x$ and $y$ are the spatial coordinates. Students can find the partial derivative in two ways: finding the slope (rise over run) at the given point on the surface and along the direction that holds $y$ constant on the surface or evaluating the ratio $(\Delta h / \Delta x)$ along the direction that holds $y$ constant on the matching contour map. Students used the surfaces during their prior middledivision physics courses, but they were not directly used in the Energy and Entropy course, nor were students specifically asked to explore thermodynamic functions using them.

\section{METHODS}

Like many other departments [33], Oregon State University's physics department has as an explicit programmatic learning outcome that students should "Demonstrate the ability to translate a physical description to a mathematical equation, and conversely, explain the physical meaning of the mathematics, represent key aspects 
of physics through graphs and diagrams, and use geometric arguments in problem-solving" [34]. To help with this learning objective, many of the upper-division faculty choose to use active-engagement classroom tasks which may require students to extract information from hands-on manipulatives and/or present information in multiple representations. We also ask students in class to solve problems that may be open ended or ambiguous in some way so that they experience a need to translate between a description of the physical situation and other representations. As a research group, we believe it is valuable to interview students using similar tasks. In these cases, our research intent is to give rich descriptions of the broad range of strategies that students employ when solving these problems and identify those places in their problem solving where they are having trouble, rather than to try to quantify particular behaviors or compare populations.

We conducted individual semistructured interviews with eight middle-division physics students who were enrolled in the Paradigms in Physics series at OSU. The participants were selected on a voluntary basis without any incentive. The interviews took place after all relevant instruction. Each interview lasted for about an hour. All interviews were videotaped using a digital camcorder.

Because the main purpose of this study is to identify the nature of the various problem-solving strategies used by middle division students, we did not select participants based on ability or grades. Our IRB protocol does not allow us to collect demographic information. All students were enrolled in OSU's Paradigms in Physics program, which, as described above, is an atypical example of upper-level physics: students in physics departments with more traditional methods of upper-division instruction may have different experiences and abilities than those who participated in this study. The reader is encouraged to exercise suitable caution in generalizing the results of this study.

\section{A. Interview task}

The interviews were conducted based on a set of predetermined questions and were structured in four stages. In the first stage, the participants were given a contour map of internal energy as a function of temperature and volume, $U(T, V)$, and a numerical table of temperature, volume, and constant pressure as shown in Fig. 3. The prompt asked the students to evaluate the partial derivative $(\partial U / \partial T)_{P}$ at a single specified point. Except for some minor clarifications, students were not interrupted or given any other prompts during this stage. In the second stage, if a student did not express the required partial derivative in terms of other computable partial derivatives, they were asked to write $(\partial U / \partial T)_{P}$ in terms of the other partial derivatives $(\partial U / \partial T)_{V},(\partial U / \partial V)_{T}$, and $(\partial V / \partial T)_{P}$. In the third stage, if a student was still unable to derive the expression, the student was prompted to use a tree diagram to express an appropriate chain rule. In the final stage, if a student still
Stage 1:

Evaluate $\left(\frac{\partial U}{\partial \boldsymbol{T}}\right)_{\boldsymbol{P}}$ at $\boldsymbol{P}=\mathbf{1 0}$ atm., $\boldsymbol{T}$
\begin{tabular}{|c|c|c|}
\hline $\boldsymbol{P ( \mathbf { a t m } . )}$ & $\boldsymbol{T}(\mathbf{K})$ & $\boldsymbol{V}\left(\mathbf{c m}^{\mathbf{3}}\right)$ \\
\hline 10 & 360 & 2.15 \\
\hline 10 & 370 & 2.32 \\
\hline 10 & 380 & 2.49 \\
\hline 10 & 390 & 2.67 \\
\hline 10 & 400 & 2.86 \\
\hline 10 & 410 & 3.05 \\
\hline 10 & 420 & 3.25 \\
\hline 10 & 430 & 3.47 \\
\hline 10 & 440 & 3.69 \\
\hline
\end{tabular}

Stage 2:

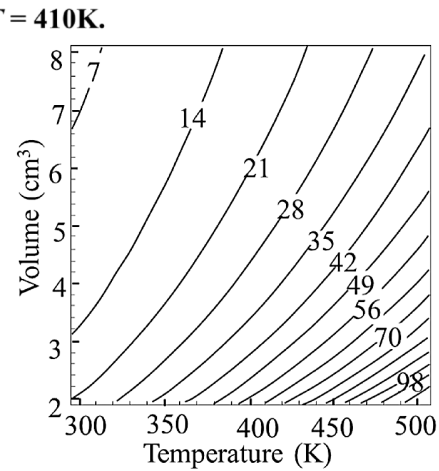

$U(V, T)$

$$
\text { Write }\left(\frac{\partial U}{\partial T}\right)_{P} \text { in terms of }\left(\frac{\partial U}{\partial T}\right)_{V},\left(\frac{\partial U}{\partial V}\right)_{T} \text {, and }\left(\frac{\partial V}{\partial T}\right)_{P} \text {. }
$$

Stage 3:

Could you use the tree diagram method to express $\left(\frac{\partial U}{\partial T}\right)_{P}$ in terms other measurable partial derivatives?

Stage 4:

$$
\begin{gathered}
\text { Compute }\left(\frac{\partial U}{\partial T}\right)_{P} \text { from the given information, if } \\
\qquad\left(\frac{\partial U}{\partial T}\right)_{P}=\left(\frac{\partial U}{\partial T}\right)_{V}+\left(\frac{\partial U}{\partial V}\right)_{T}\left(\frac{\partial V}{\partial T}\right)_{P} .
\end{gathered}
$$

FIG. 3. The problem asked in the interviews. In stage 1, students were given a page with the figure (as shown) and the prompt was also explained verbally. In stages $2-4$, more explicit prompts were given as shown.

was unable to derive the expression, they were provided with the correct formula and asked to use it to find the partial derivative.

\section{B. Initial thematic analysis}

Once the interview problem was developed, we looked for all the possible ways in which the problem can be solved correctly. We found that it can be solved by deriving an analytical expression for the required partial derivative in terms of other partial derivatives (i.e., using the multivariable chain rule). We call this approach the analytical derivation (AD) method. The AD method can be implemented in two different ways, using either differentials or a tree diagram, which have the same mathematical underpinning. To ascertain the existence of other possible methods, we consulted a knowledge extraction expert [35]. He suggested an alternative approach to solve the problem, which we call the graphical analysis (GA) method. The GA method involves analyzing the graph, identifying the constant pressure path, and calculating the indicated partial derivative along that path. Both the AD and GA methods to solve the problem are discussed in Sec. IV D.

All the interview sessions were videotaped and transcribed. We analyzed the audio-video and the written data 
together using a thematic analysis approach [20,21]. The initial themes were constructed from the ideal solutions described above. Each individual student's strategies were first identified and compared with other strategies including the ideal strategies. Then they were grouped in a way that included all the important features within the data. The goal of the thematic analysis approach is to produce compelling explanations of the patterns that emerge and the overarching themes that connect them. This approach is more flexible than other approaches, such as grounded theory [36] and phenomenological analysis [37], yet equally powerful in generating meaningful themes and reporting rich descriptions of observed phenomena.

\section{Secondary RT analysis}

Though our thematic analysis of the data revealed the strategies that students pursued, it did not clearly describe the cause of students' difficulty with multiple representations. Our data were rich enough to support a secondary analysis, which was focused on the following subsidiary research questions stated earlier: (ii) what are the patterns in how students transform between representations? and (iii) where in the process of transformation do students have difficulty?

For each generalized theme from the primary analysis, we constructed a diagram depicting the representational transformation steps involved in the problem-solving process. We call this type of diagram a representational transformation (RT) diagram (see, e.g., Fig. 7). Thus the secondary analysis is a method of describing the subthemes that arose in the thematic analysis using the RT diagrams. In an RT diagram, each representation is enclosed in a box and the transformations between representations are indicated by one or more arrows connecting the boxes. More about the RT diagrams and various transformations is presented in Sec. V.

\section{Ideal solutions}

As indicated in Sec. IV. B, the thematic analysis revealed two general ways to solve this problem: the AD method (with two submethods) and the GA method. In this section, we discuss the ideal solutions to the problem. Both methods require reading the values of $U$ from the contour map to find a numerical answer; our analysis focused mostly on students' strategies and not on the numerical accuracy of their answers. We also asked the students to neglect units in their calculations, so that they would focus mostly on their strategies.

$A D$ method.-The required partial derivative cannot be computed directly from the graph or the table. However, one can express it in terms of other partial derivatives that are more easily computed from the given representations of the data as follows:

$$
\begin{array}{crl}
U(T, V) & d U & =\left(\frac{\partial U}{\partial T}\right)_{V} d T+\left(\frac{\partial U}{\partial V}\right)_{T} d V \\
V(T, P) & d V & =\left(\frac{\partial V}{\partial T}\right)_{P} d T+\left(\frac{\partial V}{\partial P}\right)_{T} d P \\
U(T, P) \quad d U & =\left(\frac{\partial U}{\partial T}\right)_{P} d T+\left(\frac{\partial U}{\partial P}\right)_{T} d P \\
d U=\left[\left(\frac{\partial U}{\partial T}\right)_{V}+\left(\frac{\partial U}{\partial V}\right)_{T}\left(\frac{\partial V}{\partial T}\right)_{P}\right] d T+\left(\frac{\partial U}{\partial V}\right)_{T}\left(\frac{\partial V}{\partial P}\right)_{T} d P \\
\left(\frac{\partial U}{\partial T}\right)_{P}=\left(\frac{\partial U}{\partial T}\right)_{V}+\left(\frac{\partial U}{\partial V}\right)_{T}\left(\frac{\partial V}{\partial T}\right)_{P}
\end{array}
$$

FIG. 4. The differentials submethod for deriving the analytical expression of the desired partial derivative.

$$
\left(\frac{\partial U}{\partial T}\right)_{P}=\left(\frac{\partial U}{\partial T}\right)_{V}+\left(\frac{\partial U}{\partial V}\right)_{T}\left(\frac{\partial V}{\partial T}\right)_{P}
$$

The equation above is a multivariable chain rule. In the Paradigms in Physics, students are generally introduced to two principal methods for deriving a chain rule: the differentials method (Fig. 4) and the tree diagram method (Fig. 5). However, it should be noted that other methods may also be used [10].

\section{The differentials submethod}

In the differentials method, the dependent variables in different representations are represented by functional forms, such as $U(T, V)$ from the graph, $V(T, P)$ from the table, and $U(T, P)$ from the symbolic expression of the required partial derivative. Then the total differential for each variable is found using the differentials version of the chain rule, as shown in Fig. 4. The differential $d V$ in the second expression is substituted into $d V$ in the first expression and then the resulting expression is factored and compared term by term with the third expression to "identify" the desired analytical expression for the required partial derivative.

Once the equation is derived one can approximate the individual partial derivatives from the graph and the table

$$
\left(\frac{\partial U}{\partial T}\right)_{P}=\left(\frac{\partial U}{\partial T}\right)_{V}+\left(\frac{\partial U}{\partial V}\right)_{T}\left(\frac{\partial V}{\partial T}\right)_{P}
$$

FIG. 5. Deriving the partial derivative in terms of other partial derivatives using the tree diagram submethod. 
and substitute them into the equation to find the required partial derivative as follows:

$$
\begin{aligned}
& \left.\left(\frac{\partial U}{\partial T}\right)_{P} \approx \frac{\Delta U}{\Delta T}\right|_{V=3.05}+\left.\left.\frac{\Delta U}{\Delta V}\right|_{T=410} \frac{\Delta V}{\Delta T}\right|_{P=10} \\
& \left(\frac{\partial U}{\partial T}\right)_{P} \approx \frac{49-35}{434-388}+\left(\frac{35-49}{3.78-2.58}\right)\left(\frac{3.25-2.86}{420-400}\right) \\
& \left(\frac{\partial U}{\partial T}\right)_{P} \approx \frac{14}{46}+\left(\frac{-14}{1.2}\right)\left(\frac{0.39}{20}\right)=0.07 .
\end{aligned}
$$

\section{The tree diagram submethod}

The tree diagram is a mnemonic device that can be used to encode the information in the differentials method in diagrammatic form, to express a partial derivative of a function of several variables in terms of other partial derivatives using the chain rule. In the given problem, because $U$ is a function of $T$ and $V$ as depicted in the graph, one can branch out the differential $d U$ into the differentials $d T$ and $d V$. Similarly, because $V$ is a function of $T$ and $P$ as seen in the table, one can branch out $d V$ into $d T$ and $d P$. Since $T$ is a function only of itself, the two $T$ s can be connected with an arrow as shown in Fig. 5. For each arrow, there is a partial derivative of the variable at its tail with respect to the variable at its head with the adjacent variable at the same horizontal level held constant. To compute a partial derivative, one adds up the contributions from all the possible paths that connect the dependent variable at the top $(d U)$ to the independent variable at the bottom $(d T)$. The partial derivatives near each arrow are multiplied while moving from one differential to another along a specific path. For example, while going from $d U$ to $d T$ along the right-hand path, $(\partial U / \partial V)_{T}$ is multiplied by $(\partial V / \partial T)_{P}$, whereas for the left-hand path, $(\partial U / \partial T)_{V}$ is multiplied to 1. In order to find an expression for the desired partial derivative, one adds the contributions from all the possible paths that one can follow to get from $d U$ to $d T$.

GA method.-Another solution method for this problem involves use of the table and graph without an explicit chain rule formula. All the values of temperature and volume in the given table correspond to the same pressure value $(P=10 \mathrm{~atm}$.), so it is possible to plot the corresponding values of $T$ and $V$ on the given graph to create a path along which the pressure is constant. Then $(\partial U / \partial T)_{P}$ can be approximated by computing the ratio $\Delta U / \Delta T$ in the neighborhood of $(T, V)=(410,3.05)$ along the constant pressure path. Figure 6 depicts this path of constant pressure (the curve connecting the dots). Any pair of close neighboring values of $U$ and $T$ along the path can be used to estimate the desired ratio. In the example depicted, the dots immediately adjacent to $(T, V)=(410,3.05)$ are used. It is important to note that this path of constant pressure is very close to, but not exactly the same as, the

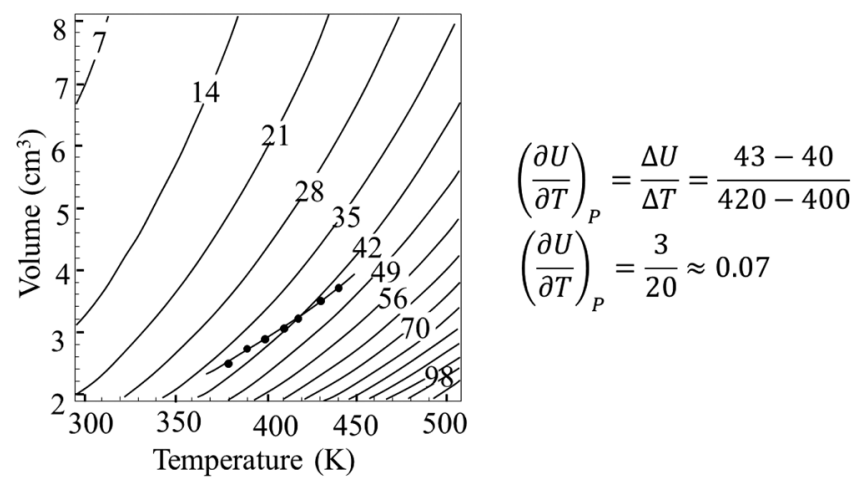

FIG. 6. One possible method for evaluating the desired partial derivative using graphical analysis strategy.

$U=42$ line. This strategy was chosen by one of the knowledge extraction experts.

\section{RESEARCH FINDINGS}

Our initial analysis revealed that students pursued either the GA approach or the AD approach. Furthermore, those who pursued the $\mathrm{AD}$ approach used either the differentials method or the tree diagram method. We found exemplars of each of the expected solution methods. No new methods arose in the student data. However, we saw significant variation in the details of how each method was implemented. Our secondary analysis focused on how students dealt with the given representations to solve the problem.

In particular, we were interested in students' transformation of representations during the problem-solving process. The representations that are discussed in this study are external representations that are expressed by the students either verbally or in written form. The partial derivative problem shown in Fig. 3 contains three principal representational classes: symbolic, numerical, and graphical. Although the initial interview prompt involved only the three representational classes, we also included a fourth class in our analysis, the diagrammatic representation, because one of the solution methods of interest involved drawing a chain rule diagram.

We observed two broad categories of representational transformations, namely, intraclass transformations and interclass transformations. In the former, the transformation occurs between two representations of the same class, such as from one symbolic expression to another symbolic expression. In the latter, the transformation occurs between two representations of different classes, such as from a numerical representation, e.g., a data table for the values of $P, T$, and $V$, to a symbolic functional form, e.g., $V(T, P)$. We also observed three common transformation phenomena in students' strategies, which we called translation, consolidation, and dissociation.

Translation is a process in which one representation is transformed into another. It may be either an intraclass or 
an interclass transformation. For example, transforming $(\partial U / \partial T)_{P}$ into $U(T, P)$ is an intraclass translation and transforming the values $V=3.05 \mathrm{~cm}^{3}, T=410 \mathrm{~K}$, into a point on a graph is an interclass translation.

Consolidation is a process in which a student transforms two or more representations into a single representation. Because each individual representation may carry a specific piece of information, consolidation involves not only the transformation of multiple representations into one, but also the processing of multiple pieces of information through one or more mathematical operations that may have varying degrees of sophistication. For example, consolidation of multiple differential expressions that carry separate pieces of information into a single partial derivative equation involves substitutions and comparison of coefficients, as shown in Fig. 4. This type of conversion may involve either an intraclass, interclass, or both types of transformations.

Dissociation is a transformation process in which one representation is expanded into two or more representations. For example, expressing $(\partial U / \partial T)_{P}$ as $\Delta U / \Delta T$ and the constant pressure constraint is a dissociation of the partial derivative representation. Similar to consolidation, this type of conversion may involve either an intraclass, an interclass, or both types of representations.

In the sections below, we document various analytic (AD) and graphical (GA) strategies that students used during the interviews. We also discuss the transformation phenomena associated with the strategies using a new analysis flowchart, the RT diagram. In Table I, we give an overview of the strategies each student used during each stage of the interview to help the reader stay oriented to the overall flow of the analysis.

TABLE I. The strategies used by each student to solve the partial derivative problem. Strategies in bold were completed correctly, and arrows indicate when students switched from one strategy to another. GA — graphical analysis approach; AD—analytical derivation approach. DF — differentials submethod; TD — tree diagram submethod; CCR - cyclic chain rule submethod. *-interviewer asked, "how else would you solve the problem?" or "is there any alternative way you can solve the problem?" $†$-after the interviewer asked, "can $U$ be expressed as the function of only two variables instead of three?" $\checkmark$-correctly computed the individual partial derivatives from the table and the graph.

\begin{tabular}{|c|c|c|c|c|}
\hline \multirow[b]{2}{*}{ Student } & \multicolumn{4}{|c|}{ Student strategy } \\
\hline & Stage 1 & Stage 2 & Stage 3 & Stage 4 \\
\hline Student 1 & $\mathbf{G A} \stackrel{*}{\rightarrow}$ No & $\mathrm{CCR} \rightarrow \mathbf{D F}$ & TD & $\checkmark$ \\
\hline Student 2 & $\mathrm{GA} \rightarrow \mathbf{G A} \stackrel{*}{\rightarrow} \mathrm{AD}$ & $\mathrm{DF} \rightarrow \mathrm{TD}$ & $\mathbf{T D}^{\dagger}$ & $\checkmark$ \\
\hline Student 3 & $\mathrm{AD} \rightarrow \mathrm{GA} \rightarrow \mathbf{G A} \stackrel{*}{\rightarrow} \mathrm{AD}$ & $\mathrm{AD}$ & TD & $\checkmark$ \\
\hline Student 4 & $\mathrm{AD} \rightarrow \mathrm{GA}$ & $\mathrm{CCR} \rightarrow \mathrm{TD}$ & $\cdots$ & $\checkmark$ \\
\hline Student 5 & $\mathrm{AD} \rightarrow \mathbf{G A}$ & TD & $\ldots$ & $\checkmark$ \\
\hline Student 6 & $\mathrm{AD} \rightarrow \mathrm{GA} \rightarrow \mathrm{AD}$ & $\mathrm{TD} \rightarrow \mathrm{DF}$ & $\cdots$ & $\checkmark$ \\
\hline Student 7 & $\mathrm{AD}$ & CCR and DF & $\mathrm{TD}$ & $\checkmark$ \\
\hline Student 8 & $\mathrm{AD}$ & TD & $\cdots$ & $\checkmark$ \\
\hline
\end{tabular}

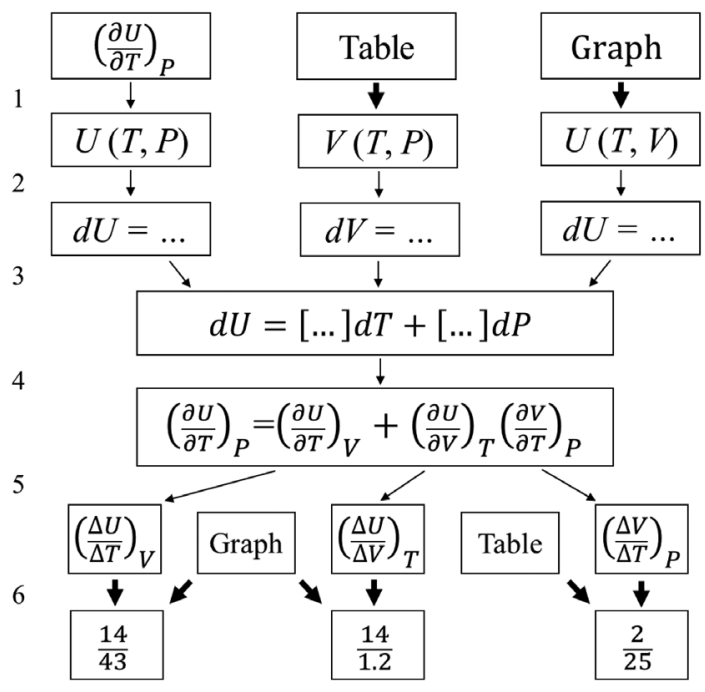

FIG. 7. An analytical derivation strategy-the differentials method. This strategy involves translation, consolidation, and dissociation.

In an RT diagram (see, e.g., Fig. 7), each representation is enclosed in a box, a translation is designated by a single arrow pointing from one box to another box, a consolidation is indicated by arrows from multiple boxes pointing into a single box, and a dissociation is depicted by multiple arrows generating from one box entering into more than one boxes. For reference purpose, we use numbers on the left side of the diagram to show the major transformation steps involved in a solution. We use a thin arrow and a bold thick arrow to indicate intraclass and interclass representations, respectively.

\section{A. AD strategy}

In an $\mathrm{AD}$ strategy, a correct derivation of the partial derivative equation is a crucial part of a correct solution. This strategy was initially pursued by six students during the first stage of the interviews. Of the six students, none solved the problem correctly during the first stage. For these students, the derivation of this equation was the most challenging aspect of their problem-solving process. These students made use of various resources, some appropriate, including the single and multivariable chain rules, and some inappropriate, including the cyclic chain rule, the ideal gas law, and the first law of thermodynamics. Not only were students unable to provide a correct solution, but they were also unable to arrive at a conclusive result. During the second and the third stages, everyone pursued the AD approach because they were provided a welldefined instruction to derive a specific equation. However, only five students completed a correct $\mathrm{AD}$ and the other three remained inconclusive or incomplete. In the fourth stage of the interviews, the students who were unable to derive the equation were provided the equation. Once the students had the equation, all of them successfully 
computed the individual partial derivatives and thus the required partial derivative using the graph and the data table together. Below, we present examples of the different ways students manifested the AD strategy.

\section{AD strategy 1: The differentials submethod}

This method involves deriving the multivariable chain rule using total differentials. A correct solution using the differentials method is discussed in Sec. IV D 1. The representational transformations involved in this type of strategy are depicted in Fig. 7.

From the representational transformation perspective, it is first necessary to translate the desired partial derivative expression, the data table, and the contour graph into functional forms as depicted in step 1 of Fig. 7. These translations involve both intra- and interclass transformations, as indicated by the fine and bold arrows, respectively. Next, in step 2 the functional forms are transformed into total differentials. The next step is to consolidate the differentials into a single total differential whose coefficients are combinations of partial derivatives (step 3). In step 4, the desired partial derivative is "identified" as the coefficient of the first term of this consolidated total differential. Then each of the partial derivatives in this equation must be translated into corresponding ratios of differences in step 5. The ratio expressions, along with the graph and the table, are consolidated into the numerical ratios as shown in step 6 of Fig. 7. To calculate a final numerical answer, the numerical ratios are multiplied and/ or summed (not shown in the figure).

None of the students who pursued the differentials method were able to derive the partial derivative expression correctly. During the first stage, some students correctly transformed the given functional form $U(T, V)$ into the correct total differential, whereas others invoked an incorrect functional form $U(T, V, P)$ and transformed it into the total differential in terms of three independent variables. However, none transformed the table and the given partial derivative expressions into their corresponding functional forms.

During the second stage of the interviews, students who pursued the differentials method transformed the given partial derivatives in terms of the corresponding functional forms either explicitly or implicitly. They also correctly invoked the corresponding total differentials either directly from the given partial derivatives or through the functional forms. Despite invoking all the correct total differentials, these students were unable to consolidate the differentials. They considered various cyclic chain rule expressions in order to consolidate the total differentials without any success. Below, we present an example of a student who unsuccessfully attempted the AD strategy during the first and second stages of the interview.

As shown in Fig. 8, student 4 correctly transformed the given functional form $U(T, V)$ into the total differential.

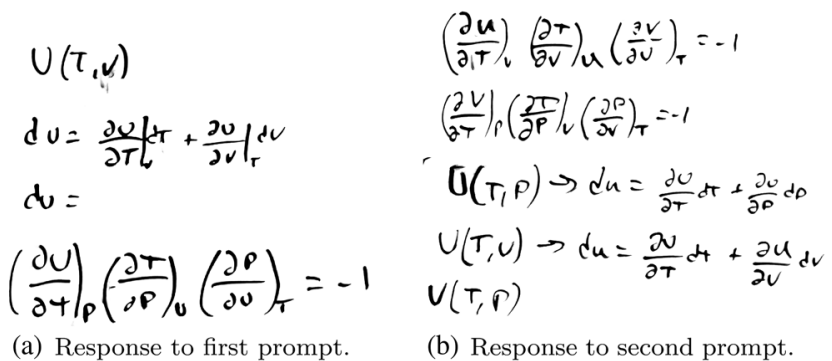

FIG. 8. Student 4's AD strategy. (a) During the first stage, he failed to transform the given representations into functional forms. (b) In the second stage, he failed to consolidate the differentials into a single equation.

However, he did not transfer the other representations to the functional forms. He invoked a cyclic chain rule, but did not find it useful. He gave the reason as,

Student 4: The reason I don't think it is useful is because $I$ want a derivative of $U$ with respect to $T$ holding pressure constant, but... I know from the total differential of $U$, I have derivatives of $U$ with respect to $T$, holding volume constant and vice versa and none of that is in the cyclic chain rule. So, that makes me think it's not going to be useful. [Pause] Actually, I guess, I'm little bit stuck. I real-, I really don't know where to go. Is this [referring to $U(T, V)$ ] saying this graph is a plot of the internal energy?

After realizing what the level curves represented, he shifted his focus towards a GA strategy. After attempting the GA strategy inappropriately, the interviewer gave him the second prompt, in response to which he invoked multiple cyclic chain rule expressions. Finding him stuck on the cyclic chain rule expressions, the interviewer asked him if he could use an alternative approach. In response, he transformed the given partial derivatives into functional forms, which were then transformed into total differentials as shown in Fig. 8. Despite the correct transformation, he failed to consolidate the total differentials into the partial derivative expression. Instead, he expressed the given partial derivatives as branch diagrams and then derived the expression successfully by pursuing the tree diagram submethod. This is discussed in more detail in the next section.

During the second stage of the interviews, the students who chose the second AD strategy, the tree diagram method discussed in the next section, successfully expressed the given representations into branched functional forms. This indicates that the students' failure to transform the given representations into the corresponding functional forms during the first stage might not be due to an inability to see or interpret the given representations as functional forms. It could, instead, be due to an inability to deal with the multiple representations and the openness of the prompt. 


\section{AD strategy 2: The tree diagram submethod}

The tree diagram submethod is an alternative $\mathrm{AD}$ strategy with the same mathematical underpinning as the differentials submethod, namely, the multivariable chain rule. An ideal tree diagram solution is presented in Sec. IV D 2. In this submethod, one first translates the given representations into functional forms with the dependent variables branching out into their independent variables as shown in step 1 of Fig. 9. As in the differentials method, this conversion involves both intra- and interclass transformations. In the second step, the functional forms are then consolidated into a single tree diagram representation, which involves intraclass transformations. The consolidation process involves several ministeps which are not shown in separate diagrams, such as connecting dependent and independent variables, writing partial derivatives, and identifying and adding the paths. These steps are discussed in detail in Sec. IV D 2. Once the proper tree diagram is constructed, it is then translated into the partial derivative equation as seen in step 3. After deriving the chain rule equation, the rest of the steps are exactly the same as the corresponding steps in the differentials submethod as discussed in the previous section.

Students were introduced to the tree diagram submethod in one of the Paradigms in Physics courses. As seen in Table I, five students pursued this submethod during the second stage of the interview and three during the third stage. Of all eight, five students implemented this method successfully. However, three were unable to implement it

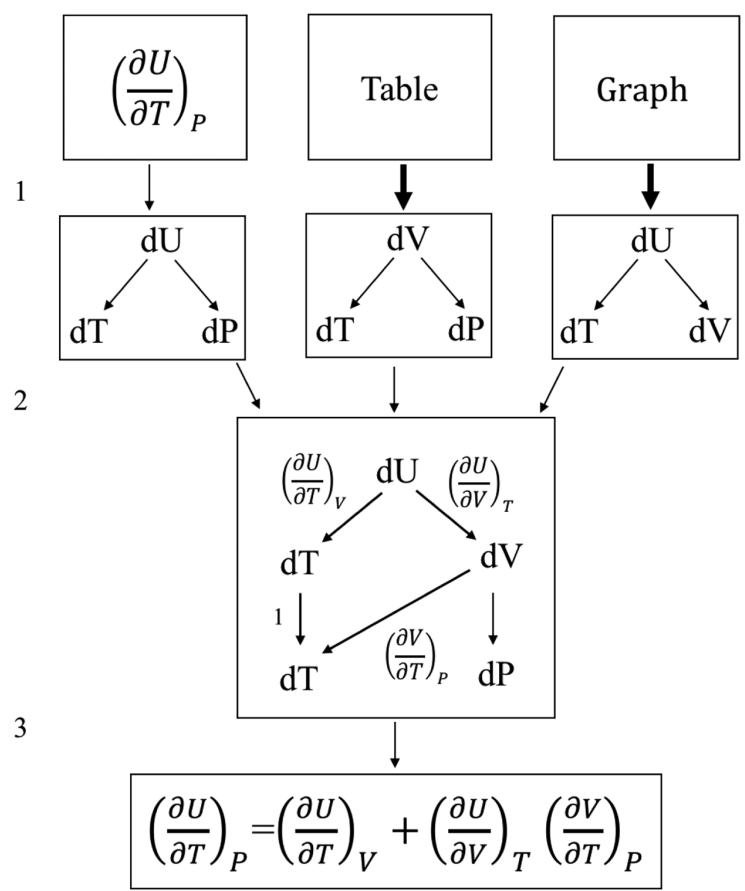

FIG. 9. An AD strategy-the tree diagram submethod. This strategy involves simple translation, consolidation, and dissociation. properly due to difficulty with either the initial translation of the given representations into branched functional forms (step 1), the consolidation of the branched functional forms into the tree diagram (step 2), or the translation of the tree diagram into the partial derivative equation (step 3). Below, we discuss two cases that depict the ways in which students implemented the tree diagram submethod.

As shown in Fig. 10, student 4's tree diagram aligned closely with the ideal solution, manifesting almost all the transformation steps depicted in Fig. 9. Unlike student 4, student 1 struggled with one of the steps, but eventually was able to complete his solution with some scaffolding, as discussed below.

When student 1 was explicitly asked to use the tree diagram, he said that since he used the tree diagram only a couple of times in his previous classes, he remembered it only vaguely. When the interviewer asked him to try it out, he started by drawing the diagram shown in Fig. 11(a) based on the correct functional relations $U(T, P), U(T, V)$, and $V(T, P)$. It was clear that student 1 had no issue with the initial translation of representations.

After translating each of the given representations into branch forms that are analogous to the symbolic functional forms, student 1 identified the partial derivative that he needed to determine, as well as the partial derivatives that he could compute from the graph and table. Then student 1 correctly translated the functional relations into the tree diagram shown in Fig. 11(a). However, he did not at first connect the variable $T$ on the second and third levels of the diagram. Student 1 then incorrectly wrote down the required partial derivative in terms of only the two partial derivatives corresponding to the right path. The student's incomplete tree diagram appears to have led to some

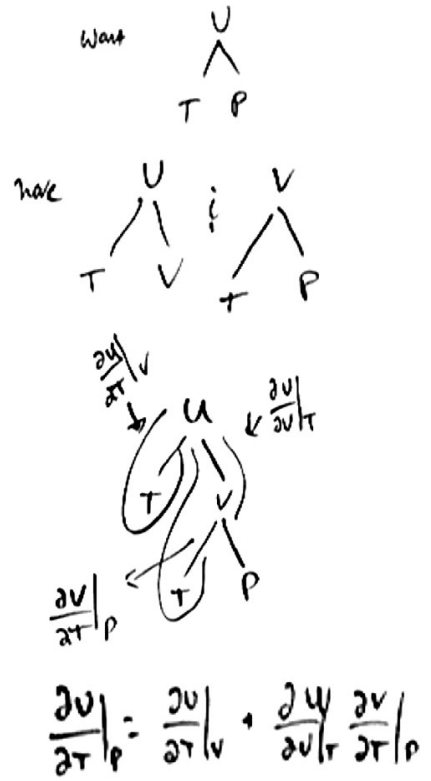

FIG. 10. Student 4's tree diagram approach to derive the partial derivative equation. 

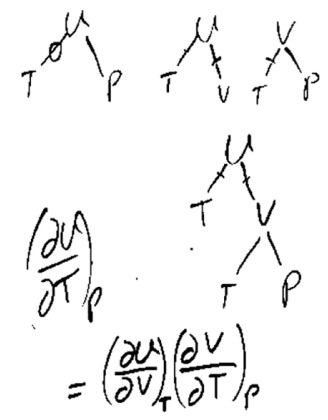

(a) Initial response.

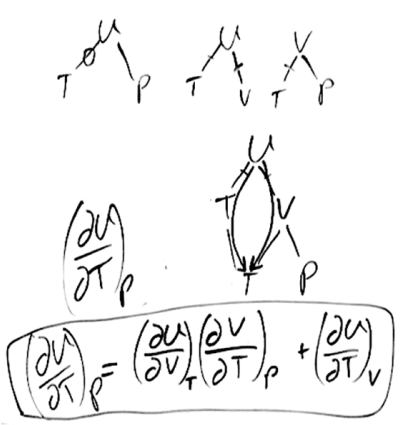

(b) Final response.
FIG. 11. Snapshots of student 1's tree diagram method. Student 1 added the paths in the tree diagram and the extra term in the PD expression after the interviewer's intervention.

difficulty with the translation process, leaving him with an incomplete expression for the partial derivative.

At this point, the interviewer recognized the missing step in student 1's transformation process and intervened by pointing at $U$ on the top and $T$ on the bottom of the tree diagram and said:

Interviewer: How many ways you get from here to here? Student 1: Oh and then I would also, I need, um, let's see... I feel like I should add this one [referring to the previous expression] plus $(\partial U / \partial T)_{V}$. Ways to get from here to here... Yeah, so I'm adding this path to this path, where this path is this chunk and this path is that chunk.

In the above excerpt, student 1 referred to the two paths that connect $U$ and $T$ on the top and the bottom of the tree diagram respectively. In response to the interviewer's targeted guidance, student 1 corrected his translation process and added the missing partial derivative to get the correct expression as shown in Fig. 11(b). Once he derived the required expression, he computed the individual partial derivatives without any issues. Not all the students who pursued the tree diagram method were able to complete it, but those who implemented it successfully were able to derive the required equation more easily than the differentials method. The tree diagram method seems to provide an elegant way to organize the variables and derivatives. Thus this method seems to reduce the effort required to connect several dependent and independent variables involved in the analytical derivation easing the transformation processes.

\section{Inappropriate and irrelevant $A D$ strategies}

During the first stage of the interviews, students manifested a number of inappropriate and inconclusive $\mathrm{AD}$ strategies. As stated earlier, they invoked various facets of mathematical and physical knowledge, such as the cyclic chain rule, differentials, the ideal gas law, and the first law of thermodynamics. Although four students attempted the differentials method to different extents, none were able to implement it correctly. Even after the interviewer's prompt to derive an expression for the partial derivative in terms of other partial derivatives, two students were still unable to arrive at a correct expression. Below we present an example of an inappropriate and inconclusive AD strategy. We chose this example to depict the types of transformation that are generally seen in these types of $\mathrm{AD}$ strategies. We also show the crucial transformation processes that students often fail to execute in $\mathrm{AD}$ strategies.

In response to the first prompt, student 3 initially wrote down $(\partial U / \partial T)_{P}$. Then he looked at the table and realized that it had the variables $P, T$, and $V$. After a momentary pause, he invoked the ideal gas law $P V=N k_{B} T$ and continued:

Student 3: Although I don't think we were given this as an ideal gas. No. [Shifting focus towards the graph] I would want to hold the volume constant and just look at the line $[V=3.05]$, the change around that point $[T=410]$.

Because the problem did not state whether or not the system was an ideal gas, he did not pursue this strategy further and shifted focus to the graph. He drew a $V=3.05$ line and described how he would find the ratio $\Delta U / \Delta T$ along that line. However, he later realized that he needed to compute the partial derivative along a constant pressure path and thus that the ratio along the $V=3.05$ path would not be appropriate. He correctly drew the constant pressure path and computed the required partial derivative.

When the interviewer pressed for an alternative approach, student 3 invoked two cyclic chain rules. However, he did not find them useful for solving the problem. Although the student identified that the problem did not indicate whether or not the system could be treated as an ideal gas, he returned to the ideal gas law and attempted to translate the constraint into a different representation so that he could translate it on the graph to find the constant pressure path. As seen in the right side of Fig. 12 in steps 1 and 2, student 3 translated the tabular representation to the symbolic representation, using the ideal gas law equation, which resulted in a correct but unhelpful equation $V=N k_{B} T / P$. As a consequence he did not find the strategy productive for this purpose and thus found it pointless to translate the resulting equation into a graphical representation. In this strategy, student 3 failed to translate the constraint to the graph because of the inappropriate intermediate process. Because the student was unable to conclude his solution, the interviewer moved on to the second stage of the interview.

In response to the prompt in the second stage, student 3 proposed a few other inappropriate strategies, including the first law of thermodynamics and Maxwell relations. The interviewer then asked him to try the tree diagram 


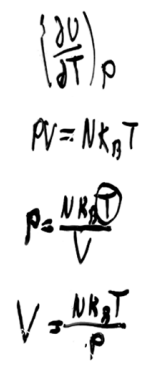

(a) Initial response.

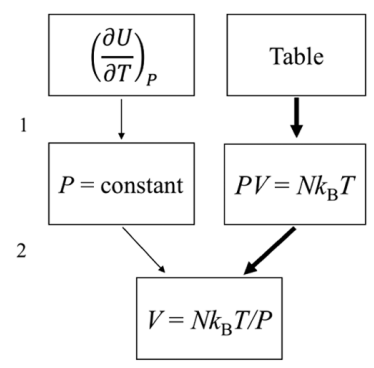

(b) Representational transformation diagram.
FIG. 12. Student 3's unproductive analytical derivation strategy. He invoked the ideal gas law and rearranged it.

method. However, his inability to translate the given representations into functional form persisted even after the interviewer reminded him about the tree diagram. Student 3 incorrectly branched $d U$ into three total differentials $d P, d V$, and $d P$ (he meant $d T$ ), clearly showing his failure to realize the functional forms of the given representations. He engaged in the method for awhile without much progress and finally gave it up, confessing that he did not remember the method. As in the previous situation, he again failed to analyze the variables in the given representations, a crucial step for initiating the tree-diagram submethod. In this example, student 3 moved back and forth between several mini strategies invoking both relevant and irrelevant knowledge bases and transformations of representations.

\section{Summary of AD strategies}

Often, thermodynamics problems involving analytical derivation pose challenges to students even when the problems involve only algebraic representations. Moreover, the problem presented in this study seemed to pose an extra layer of complexity to the $\mathrm{AD}$ process due to the presence of multiple representations. Because the problem contained multiple representations, the $\mathrm{AD}$ strategy not only required coordinating multiple facets of mathematical concepts and procedures, but also finding links between various representations. The students who pursued $\mathrm{AD}$ strategies first realized that they could not compute the required partial derivative directly from the given representations, so they needed to find an expression in terms of other directly computable partial derivatives. In order to find the correct expression, they needed to carry out a variety of transformations: identify dependent and independent variables, translate them into functional forms, write differentials, find connections between the expressions, and consolidate them into a single expression. However, none of the students were able to complete the transformation process during the first stage of the interview (i.e., without intervention from the interviewer).

Only a few of the students who pursued an AD strategy manifested some level of difficulty with translation and dissociation, but the majority showed considerable difficulty with the consolidation process. One possible reason is that consolidation involves multiple mathematical operations with varying degrees of sophistication. The consolidation process was difficult in both the differentials and the tree diagram submethods. Despite successfully translating the given symbolic, numerical, and graphical representations into functional forms and further translating them into differentials, most of the students who chose the differentials method were unable to consolidate them into a single equation. Most attempted the consolidation process by invoking and implementing the cyclic chain rule, which was not productive in the given context. The underlying difficulty with the consolidation of representations was a failure to recognize what must be substituted from one equation into another so that coefficients can be compared to get the desired equation.

Similarly, the students who chose the tree diagram method were either unable to consolidate the branched out functional form into a single tree diagram or to consolidate the tree diagram into the desired equation. Their failure to consolidate the representations was mostly due to an inability to implement the underlying rules of the tree diagram method. Most students used this method only at the interviewer's prompt.

Some students invoked inappropriate or irrelevant physics and mathematics knowledge, such as the ideal gas law, the first law of thermodynamics, and the cyclic chain rule while attempting an analytical derivation. These students did not perform all the necessary translations; instead, they unsuccessfully attempted to translate and/or consolidate the invoked representations. Even though many students were unable to execute an AD strategy, they had little difficulty computing the other partial derivatives from the table or the graph when they were provided the correct partial derivative equation. This implies that students' greatest difficulty was with the multiple representations and transformations associated with the problem.

\section{B. GA strategy}

In the GA strategy, drawing or identifying a correct constant-pressure path is a crucial part of a correct solution. As depicted in Table I, a total of six students pursued the GA strategy during the first stage of the interviews. Although two students chose this strategy in the beginning, the other four pursued it after failing with the AD strategy. Of the four students, two used the strategy correctly and the other two did not use it correctly. Below we discuss the three general ways students manifested the GA strategy in the interviews. 


\section{GA strategy considering the constraint}

An ideal GA strategy involves computing the required partial derivative along a constant pressure path, as depicted in Fig. 6. The RT diagram for this method is depicted in Fig. 13. The first step involves consolidating the partial derivative expression and the given numerical values of $T$ and $V$ into the data table. In the second step, the data table and the graph are consolidated into a constant pressure path on the graph and the partial derivative expression is translated into the ratio expression. Finally, the ratio and the constant pressure graph are consolidated into the ratio of numbers.

Four interviewees realized that it is necessary to identify a path of constant pressure in order to evaluate the required partial derivative directly from the graph. These students drew at least two points on the graph to form the path corresponding to the given constant pressure $(P=10 \mathrm{~atm})$. Below, we discuss a representative GA strategy considering the constant pressure path.

Student 1 initially approached the GA method incorrectly, but he quickly realized the correct process and manifested all the steps that are depicted in Fig. 13. Student 1 planned to compute the partial derivative using the table. In order to find the partial derivative, he first needed to find $\Delta U$ and $\Delta T$, but he realized that the table does not have any information about energy. In addition, he was not initially sure what each level curve on the contour map is intended to represent, perhaps because the numbers on the level curves do not have units. He first translated the given values of $P$ and $T$ into the table and then translated the corresponding $T$ and $V$ values to the graph. He also realized that the pressure is constant, but he was not able to consolidate either the graph or the table with the ratio due to his failure to interpret what the table and the graph represent. He asked the interviewer,

Student 1: [Pointing over the level curves] Um, what are these numbers?

Interviewer: So there is a caption below the figure. Student 1: Oh internal energy $U$ as a function of T. Oh, okay, I see... Ah, alright, I understand now.

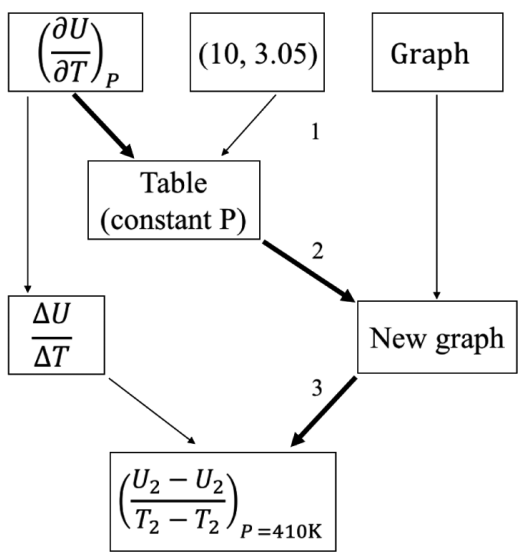

FIG. 13. The RT diagram for an ideal GA strategy.

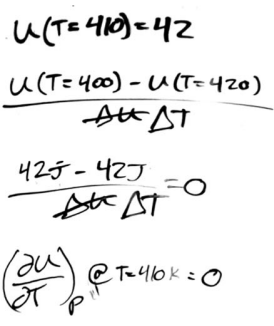

(a) Written response.

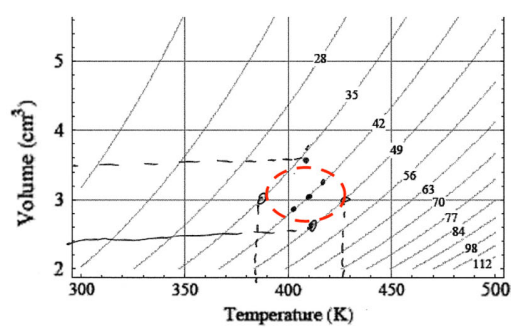

(b) Work on graph.

FIG. 14. Student 1's GA strategy for solving the problem. The dashed oval has been added to highlight the path of constant pressure that student 1 identified.

Once the interviewer pointed out the label of the graph, the functional form $U(T, V)$, student 1 quickly realized that the level curves represent the internal energy and immediately figured out how to find the required partial derivative by determining the ratio of $\Delta U$ and $\Delta T$. Although he unintentionally wrote the denominator as $\Delta U$, he later realized that the correct denominator would be $\Delta T$ as seen in Fig. 14(a). He also realized that in order to find the required partial derivative, it is necessary to use points along the constant pressure path. To find the path, he plotted three points corresponding to $P=10$ atm in the neighborhood of $T=410 \mathrm{~K}$ as shown in Fig. 14(b). Given the resolution that he was using for his points on the graph, he then found that the points lie on $U=42$, so he decided that the change in energy would be 0 . The above strategy manifested by student 1 is close to an ideal GA strategy.

As in the RT diagram shown in Fig. 13, student 1 successfully dissociated the given symbolic representation of the partial derivative into two new, distinct representations: the derivative as a ratio of small changes and the path of constant pressure. Then he consolidated the partial derivative expression and the given values of $P$ and $V$ into the table, which was then consolidated with the graph to produce a constant pressure path. Finally, he consolidated the ratio and the graph into a ratio of numerical values extracted from the graph as in step 3.

\section{GA strategy without considering constraint}

Although the problem asks for the partial derivative at a specific (constant) pressure, three students who pursued the GA strategy, including student 1 and student 3 in the previous examples, did not initially utilize the constant pressure condition while solving the problem. However, they later realized their error and corrected their strategy. Here, we present the strategy of student 2, who initially failed to realize that it was necessary to choose a path of constant pressure.

In response to the given problem, student 2 first wrote $\partial U /\left.\partial T\right|_{P}$ and then marked three dots, one at $U=42$ corresponding to $(T, V)=(410,3.05)$ and the other two in the neighborhood of that point along a horizontal line. He 
found the ratio of $\Delta U$ and $\Delta T$ using the two neighboring points. However, student 2 soon realized that the partial derivative he found above was not a correct one because the path he chose was not a constant pressure path.

Student 2: [Referring to the information given in the problem] I'm not looking at this right. This right here is not necessarily labeled ri-[ght] or how I would expect it to be labeled.

Student 2 realized that he was not interpreting the problem correctly, so he discarded the above work and corrected his strategy just as student $1 \mathrm{did}$ in the previous example. He recognized that he needed to find the partial derivative along a constant pressure path, thus he now plotted $(T, V)=(410,3.05)$ and then two other points in its neighborhood from the table. Student 2 also realized that since all the points on the table were at $P=10 \mathrm{~atm}$, the curve passing through the three points he just drew formed a constant pressure path. As with student 1, student 2 found that the constraint path overlapped the contour $U=42$, so he determined $\Delta U$ and thus the partial derivative to be zero. Although a few students needed intervention from the interviewer to recognize the constraint, it was a spontaneous realization in student 2's case.

As shown in Fig. 15, student 2 first matched the given values of temperature and pressure on the table. Then he translated the corresponding values of temperature and volume on the graph. Finally, he consolidated the graph and the ratio. Although he translated the given partial derivative into a ratio, he failed to dissociate it into two separate entities and thus overlooked the constraint. However, once he realized the constant pressure constraint, the transformation paths were similar to the one depicted in Fig. 13.

\section{Inappropriate $G A$ strategies}

Incorrect graphical analysis strategies included students using inappropriate graphical features, such as the slope of a contour line, to evaluate $(\partial U / \partial T)_{P}$. For example, in response to the initial prompt, student 4 drew a vertical line corresponding to $T=410 \mathrm{~K}$ and a horizontal line corresponding to $V=3.25 \mathrm{~cm}^{3}$, then looked for the point at which the lines intersected. When the interviewer asked him if that would give the required partial derivative, he responded

Student 4: I guess, I put that because I don't really, don't understand the question and I didn't know what to do and so I just thought maybe the intersection is the answer but ...

Student 4 did not know what exactly he was supposed to do, so he just applied the given information (the values of $T$ and $V$ ) to draw the horizontal and vertical lines on the graph. He admitted that he did not know how to find the required partial derivative because unlike a traditional

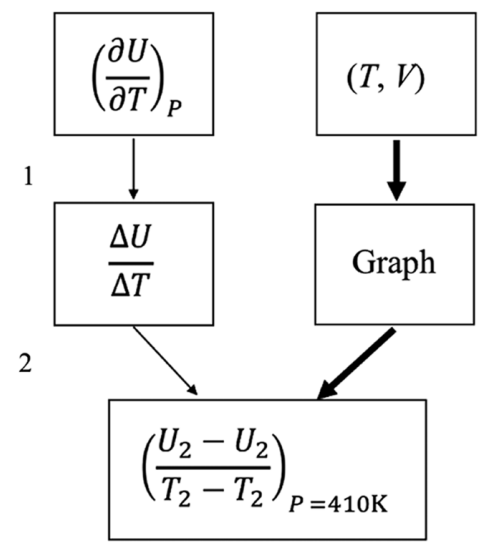

FIG. 15. GA strategy without considering constraint.

thermodynamics problem there was no algebraic function given in the problem. When the interviewer pointed out that the number he found was not actually a partial derivative, he added.

Student 4: My first instinct was... I need an answer for this problem... Um, so I just guessed, it was the intersection of the lines. But now I'm thinking, because it's a derivative and pressure isn't on this graph that it could just be the slope of the line. So now, ah, I'm, I'm, drawing a line tangent to that point and I'm gonna try to get a slope.

Student 4 was unable to come up with a reasonable GA procedure to find the partial derivative. He inappropriately evaluated the slope of a curve and thus failed to distinguish between the derivative of $U$ with respect to $T$ and the derivative of $V$ with respect to $T$. He correctly interpreted that the partial derivative should involve constant pressure, but he did not use the idea while solving with the problem. Student 4's strategy resembles student 2's strategy depicted in Fig. 15 from the RT perspective, but there is a major difference in the consolidation process between the two approaches. Despite his failure to consider the constraint, student 2 correctly consolidated the graph and found a ratio by computing the partial derivative along the constant volume line. In contrast, student 4 inappropriately consolidated the graph and the ratio by computing the slope of the tangent line to a curve, as is common when computing a derivative of a function of one variable from a graph.

\section{Summary of GA strategies}

The students who provided a correct solution using the GA strategy dissociated the given partial derivative representation into the ratio of changes and the constraint. They consolidated the constraint and the table into the graphical representation by drawing the constant pressure curve. Finally, the graph and the ratio of changes were consolidated into a numerical ratio. The GA approach seemed 
easier to implement than the AD approach. Students were more able to recognize the connections between the given representations and the use of the given constraint. Finding such connections and recognizing the constraint often involved one or more transformation processes. When students failed to execute one or more transformations, that resulted in an incomplete or inappropriate solution as documented in the case of GA strategy without considering the constraint.

Although an ideal GA strategy comprises all three transformation phenomena (as the AD strategy does), all the given representations are consolidated into a graphical representation rather than an algebraic one. For the students who failed to execute the strategy correctly, the most challenging aspect was the consolidation of the symbolic and the numerical representations of the constant pressure constraint into the graphical representation. Although some students failed to dissociate the given partial derivative, they nevertheless were able to translate the numerical representations into the graphical representation-that is, they were able to draw a line of constant volume on the graph corresponding to the given numerical value of $V$. In addition, they did not seem to have any difficulty computing the partial derivatives along the chosen path. This further implies that the difficulty was not with the partial derivative concept, but with the underlying representational transformation.

\section{CONCLUSIONS AND IMPLICATIONS}

In this study, we investigated students' strategies for and difficulty with solving a partial derivative problem with a thermodynamics context presented in multiple representations. Students'overall strategies fell into two broad categories: analytical derivation and graphical analysis. In the $\mathrm{AD}$ strategy, students derived an expression for the required partial derivative in terms of other partial derivatives using either differentials or a tree diagram. To complete the solution using this strategy, students first identified the functional form of the given partial derivative, i.e., $U(T, P)$, recognized which chain rule to use, derived the required expression, and finally computed the individual partial derivatives using the graph and the table to determine the numerical value of the required partial derivative. The GA strategy has fewer steps than the AD strategy: students plotted the constant pressure path on the given graph and then evaluated the required partial derivative graphically. The most common obstacle students had with the latter method was that they were unable to realize that they first needed to plot a constant pressure path on the graph and that with the former method was their inability to implement the chain rule correctly. The identification of the two strategies and the difficulty with each strategy together answer our primary research question. We also note that students occasionally switched between the $\mathrm{AD}$ and GA strategies, especially when they did not appear to be making progress with one strategy (see Table I). Once the students got stuck with the AD strategy, they looked for alternate way to connect the representations, for which they chose the GA strategy.

In Sec. I, we described several concepts that are required to solve advanced partial derivative problems. Comparing the responses of our interviewees to this set of concepts suggests they had little difficulty with the procedural aspects of the partial derivative and only some difficulty with the conceptual aspects, as evidenced by the fact that the majority of students successfully computed the individual partial derivatives once they had the final expression from a chain rule or the graph. Instead, the most difficult aspects for our students were in making connections between the given representations and transforming them from one class to another class. In particular, students had substantially more difficulty deriving a chain rule when the prompt was more open and involved multiple representations, as in stage 1 of the interviews, than when it was direct and involved only the symbolic representation, as in stages 2 and 3 of the interviews. The students who failed to solve the problem either got stuck or switched to other strategies involving different representational transformations, as in student 3's case, discussed in Sec. VA. These findings inspired us to analyze the students' strategies using the perspective of representational transformation (RT) focusing mostly on how students transitioned between classes of representations-symbolic, numerical, graphical, and diagrammatic-while solving the problem.

We analyzed students' strategies based on the representational transformation perspective to answer the secondary research questions. Our analysis revealed that students' inability to solve the problem was primarily due to an inability to deal with various aspects of multiple representations. Although there are a number of studies on the role of multiple representations in problem-solving in PER and RUME, only a few of them seem to have focused specifically on representational transformation phenomena in student strategies. There also seems to be a lack of specific methods for analyzing the transformation phenomena. Thus, we have developed a new type of flow chart to depict the transformations that arose in the process of problem solving, which we called representational transformation diagrams. We consider RT diagrams to be an elegant way to show the steps involved in a problem-solving process across various contexts from a representational transformation perspective.

The transitions were either within or between the classes, which we called intraclass transformation and interclass transformations, respectively. We found three distinct ways that students transformed the given representations: translation, consolidation, and dissociation. Translation is a one-to-one transformation of representations, consolidation involves combining two or more representations into a single representation, and dissociation involves breaking a single representation into more than one representation. The representational transformation phenomena answer our 
second research question about the ways students deal with the representations involved in the problem.

We found the answer to our third research question by comparing the RT diagrams for incorrect or incomplete solutions to the RT diagram for an ideal solution. The comparisons reveal that the type of transformation that proved most difficult for many students was the consolidation process. Student difficulty with the consolidation process was often due to an inability to utilize the cues presented in the problem or to invoke the mathematical tools necessary to connect different representations.

There has been a strong emphasis on using multiple representations in conceptual understanding and problemsolving in STEM and other fields [24,25,38,39]. Previous studies have shown that students often have difficulty with various aspects of multiple representations including the translation process $[2-4,24,25]$. Studies on multiple representations in K-12 and lower-division contexts have documented translations between and within different representational classes, which we referred to as interclass and intraclass transformations $[38,40]$. However, prior research has not discussed either consolidation or dissociation. We think that consolidation and dissociation are particularly important in middle- and upper-division problem solving. The participants in this study, who were middle-division students, did not seem to have as much trouble with simple translation as with consolidation and dissociation.

We found that students often responded effectively to minimal but targeted scaffolding questions, including the prompt in the second and the third phases of the interviews, which specified a major intermediate step in solving the problem analytically. This suggests that instructors might help students with complicated consolidation and dissociation processes by guiding them to identify goals such as the objective of a consolidation step in a procedure. Making students consciously aware of the three different types of representational transformation processes may therefore help them solve problems.

In this study, we implemented the RT method to analyze student strategies for solving one problem only. The method was essential to our analysis from the perspective of multiple representations, which was necessary to answer our research questions. However, we suggest that the examples we presented do not show everything that might be possible with this analysis method. We intend to utilize this method in our future research as well as in our instruction. We also anticipate that other researchers may find this method useful for analyzing student strategies for solving multirepresentation problems in other mathematical contexts.

We suggest that RT diagrams might be useful not only for analyzing data, but also for instructional purposes. For example, individual instructors might construct ideal RT diagrams for all possible solutions of in-class or homework problems. Such diagrams could then be used to identify and prepare for the particular transformations that students will end up considering. This knowledge would have value both as a means for preparing to interact with students and when identifying learning goals. Similarly, curriculum developers might benefit from RT diagrams when analyzing classroom tasks, both for describing ideal solutions and for assessing how students actually solve problems.

\section{ACKNOWLEDGMENTS}

We would like to acknowledge the OSU Paradigms group members for their thoughtful input on this manuscript. We thank the students who volunteered to participate in this study. We also thank the anonymous reviewers for their valuable suggestions and feedback. This research is supported in part by the National Science Foundation under Grant No. DUE-1323800.
[1] R. R. Bajracharya and J. R. Thompson, Analytical derivation: An epistemic game for solving mathematically based physics problems, Phys. Rev. Phys. Educ. Res. 12, 010124 (2016).

[2] J. R. Thompson, B. R. Bucy, and D. B. Mountcastle, Assessing student understanding of partial derivatives in thermodynamics, AIP Conf. Proc. 818, 77 (2006).

[3] B. R. Bucy, J. R. Thompson, and D. B. Mountcastle, Student (mis)application of partial differentiation to material properties, AIP Conf. Proc. 883, 157 (2007).

[4] N. Becker and M. Towns, Students' understanding of mathematical expressions in physical chemistry contexts:
An analysis using Sherin's symbolic forms, Chem. Educ. Res. Pract. 13, 209 (2012).

[5] M. B. Kustusch, D. Roundy, T. Dray, and C. Manogue, An expert path through a thermo maze, AIP Conf. Proc. 1513, 234 (2013).

[6] M. B. Kustusch, D. Roundy, T. Dray, and C. A Manogue, Partial derivative games in thermodynamics: A cognitive task analysis, Phys. Rev. ST Phys. Educ. Res. 10, 010101 (2014).

[7] D. Roundy, E. Weber, T. Dray, R. R. Bajracharya, A. Dorko, E. M. Smith, and C. A. Manogue, Experts' understanding of partial derivatives using the partial derivative machine, Phys. Rev. ST Phys. Educ. Res. 11, 020126 (2015). 
[8] E. Gire, T. Dray, C. A. Manogue, and D. Roundy, Representations of partial derivatives, in Proceedings of the EnFUSE Symposium, Washington, DC (AAAS, Washington, DC, 2016), https://tinyurl.com/yyfcxrsu.

[9] P. J. Emigh and C. A. Manogue, Student interpretations of partial derivatives, in Proceedings of the 2017 Physics Education Research Conference, Cincinnati, OH, edited by L. Ding, A. Traxler, and Y. Cao (AIP, New York, 2017), pp. 120-123, https://doi.org/10.1119/perc.2017.pr.025.

[10] I. W. Founds, P. J. Emigh, and C. A. Manogue, Student responses to chain rule problems in thermodynamics, in Proceedings of the 2017 Physics Education Research Conference, Cincinnati, $\mathrm{OH}$, edited by L. Ding, A. Traxler, and Y. Cao (AIP, New York, 2017), pp. 136-139, https:// doi.org/10.1119/perc.2017.pr.029.

[11] P. J. Emigh and C. A. Manogue, How students find derivatives from contour graphs (to be published).

[12] T. Dray, E. Gire, M. B. Kustusch, C. A. Manogue, and D. Roundy, Interpreting derivatives, PRIMUS 29, 830 (2019).

[13] D. Roundy, M. B. Kustusch, and C. Manogue, Name the experiment! Interpreting thermodynamic derivatives as thought experiments, Am. J. Phys. 82, 39 (2014).

[14] P. J. Emigh, R. R. Bajracharya, T. Dray, E. Gire, D. Roundy, and C. A. Manogue, Learning progressions in math and physics: An example for partial derivatives, in Proceedings of the 21st Annual Conference on Research in Undergraduate Mathematics Education (Mathematical Association of America, Oberlin, OH, 2018), pp. 1189-1196.

[15] Webpage of the Paradigms in Physics project, http:// physics.oregonstate.edu/portfolioswiki, contains a description of the Energy and Entropy course, a summary of the course content, and detailed descriptions of the activities used in the course.

[16] C. A. Manogue, P. J. Siemens, J. Tate, K. Browne, M. L. Niess, and A.J. Wolfer, Paradigms in physics: A new upper-division curriculum, Am. J. Phys. 69, 978 (2001).

[17] C.A. Manogue and K.S. Krane, The Oregon State University paradigms project: Re-envisioning the upper level, Phys. Today 56, 53 (2003).

[18] D. Roundy and M. Rogers, Exploring the thermodynamics of a rubber band, Am. J. Phys. 81, 20 (2013).

[19] G. Sherer, M. B. Kustusch, C. A. Manogue, and D. J. Roundy, The Partial Derivative Machine, in Proceedings of the 2013 Physics Education Research Conference, Portland, OR, edited by P. V. Engelhardt, A. D. Churukian, and D. L. Jones (AIP, New York, 2013), pp. 341-344, https://doi.org/10.1119/perc.2013.pr.073.

[20] V. Braun and V. Clarke, Using thematic analysis in psychology, Qual. Res. Psychol. 3, 77 (2006).

[21] G. Guest, K. M. MacQueen, and E. E. Namey, Applied Thematic Analysis (SAGE, Thousand Oaks, CA, 2012), https://dx.doi.org/10.4135/9781483384436.

[22] K. P. Browne, A case study of how upper-division physics students use visualization while solving electrostatics problems, Ph.D. thesis, Oregon State University, 2001, https://tinyurl.com/y2w3f47z.
[23] M. Zandieh, A theoretical framework for analyzing student understanding of the concept of derivative, CBMS Issues Math. Educ. 8, 103 (2000).

[24] P. B. Kohl and N. D. Finkelstein, Student representational competence and self-assessment when solving physics problems, Phys. Rev. ST Phys. Educ. Res. 1, 010104 (2005).

[25] P. B. Kohl and N. D. Finkelstein, Effects of representation on students solving physics problems: A fine-grained characterization, Phys. Rev. ST Phys. Educ. Res. 2, 010106 (2006).

[26] T. Eisenberg and T. Dreyfus, On visual versus analytical thinking in mathematics, in Proceedings of the Tenth International Conference for Psychology of Mathematics Education, London, UK (1986), pp. 153-158.

[27] S. Vinner, The avoidance of visual considerations in calculus students, Focus Learn. Probl. Math. 11, 149 (1989).

[28] M. K. Heid, Resequencing skills and concepts in applied calculus using the computer as a tool, J. Res. Math. Educ. 19, 3 (1988).

[29] D. Roundy, A. Gupta, J. F. Wagner, T. Dray, and M. Bridget, From fear to fun in thermodynamics, in Proceedings of the 2013 Physics Education Research Conference, Portland, OR, edited by P. V. Engelhardt, A. D. Churukian, and D. L. Jones (AIP, New York, 2013), pp. 42-45, https:// doi.org/10.1119/perc.2013.inv.009.

[30] J. R. Thompson, C. A. Manogue, D. J. Roundy, and D. B. Mountcastle, Representations of partial derivatives in thermodynamics, AIP Conf. Proc. 1413, 85 (2012).

[31] Surfaces Project Team, Raising calculus to the surface, http://raisingcalculus.winona.edu (2013-2016).

[32] A. Wangberg and B. Johnson, Discovering calculus on the surface, PRIMUS 23, 627 (2013).

[33] S. Pollock, S. Chasteen, M. Dubson, and K. Perkins, The use of concept tests and peer instruction in upper-division physics, AIP Conf. Proc. 1289, 261 (2010).

[34] Learning Outcomes for the BS in Physics Program, Oregon State University, https://physics.oregonstate.edu/ LearningOutcomesBS.

[35] B. R. Wilcox, M. D. Caballero, D. A. Rehn, and S. J. Pollock, Analytic framework for students' use of mathematics in upper-division physics, Phys. Rev. ST Phys. Educ. Res. 9, 020119 (2013).

[36] A. Strauss and J. M. Corbin, Grounded Theory in Practice (SAGE Publications, Newbury Park, CA, 1997).

[37] J. A. Smith, Qualitative Psychology: A Practical Guide to Research Methods (Sage, Newbury Park, CA, 2007).

[38] S. Ainsworth, The functions of multiple representations, Comput. Educ. 33, 131 (1999).

[39] D. F. Treagust, R. Duit, and H. E. Fischer, Multiple Representations in Physics Education, Ph.D. thesis, Springer, 2017.

[40] R. Lesh, T. Post, and M. Behr, Representations and translations among representations in mathematics learning and problem solving, Problems of Representation in the Teaching and Learning of Mathematics 21, 33 (1987). 